\title{
Optimum Restoration of Radial Electric Distribution Networks Using Multi-Objective Genetic Algorithms
}

\author{
Ricardo Rivas Giménez, M.Sc. ${ }^{1}$, Enrique Ramón Chaparro, D.Sc. ${ }^{2}$ \\ ${ }^{1}$ ANDE, Paraguay, ricardo_rivas@ande.gov.py, \\ ${ }^{2}$ Facultad Politécnica de la Universidad Nacional del Este (FPUNE), Paraguay, enriquerchv@fpune.edu.py
}

\begin{abstract}
The present work deals with the problem of restoration of a radial and balanced Electric Distribution System, that after an event causes the programmed disconnection, or not programmed, remains with one or several stretches of feeders without power. The load restoration methodology uses the automatic reconfiguration algorithm, establishing a new arrangement of closed and open interruptors that modifies the topology, seeking the reconfiguration for energy restoration that simultaneously optimizes two objectives: the load to be restored and the number of possible maneuvers. Both objectives are simultaneously optimized by the Genetic Algorithm, adapted for multi-objective optimization through the weighted sum of objective functions. In order to validate the proposed method, three electric distribution system were considered, two small size academics systems and one medium scale real system, belonging to the ANDE distribution system. The restoration results, obtained in a substantially reduced computational time, demonstrate the efficiency of the proposed method, which could be a useful tool in load dispatch and operation planning.

Keywords-- Radial distribution system, restoration, reconfiguration, multi-objective optimization, Genetic Algorithm.
\end{abstract}

Digital Object Identifier (DOI):

http://dx.doi.org/10.18687/LACCEI2020.1.1.97

ISBN: 978-958-52071-4-1 ISSN: 2414-6390

18 $^{\text {th }}$ LACCEI International Multi-Conference for Engineering, Education, and Technology: "Engineering, Integration, and Alliances for a Sustainable Development" "Hemispheric Cooperation for Competitiveness and Prosperity on a Knowledge-Based Economy", 27-31 July 2020, Virtual Edition. 


\title{
Optimum Restoration of Radial Electric Distribution Networks Using Multi-Objective Genetic Algorithms
}

\author{
Ricardo Rivas Giménez, M.Sc. ${ }^{1}$, Enrique Ramón Chaparro, D.Sc. ${ }^{2}$ \\ ${ }^{1}$ ANDE, Paraguay, ricardo_rivas@ande.gov.py, \\ ${ }^{2}$ Facultad Politécnica de la Universidad Nacional del Este (FPUNE), Paraguay, enriquerchv@fpune.edu.py
}

\begin{abstract}
The present work deals with the problem of restoration of a radial and balanced Electric Distribution System, that after an event causes the programmed disconnection, or not programmed, remains with one or several stretches of feeders without power. The load restoration methodology uses the automatic reconfiguration algorithm, establishing a new arrangement of closed and open interruptors that modifies the topology, seeking the reconfiguration for energy restoration that simultaneously optimizes two objectives: the load to be restored and the number of possible maneuvers. Both objectives are simultaneously optimized by the Genetic Algorithm, adapted for multi-objective optimization through the weighted sum of objective functions. In order to validate the proposed method, three electric distribution system were considered, two small size academics systems and one medium scale real system, belonging to the ANDE distribution system. The restoration results, obtained in a substantially reduced computational time, demonstrate the efficiency of the proposed method, which could be a useful tool in load dispatch and operation planning.

Keywords-- Radial distribution system, restoration, reconfiguration, multi-objective optimization, Genetic Algorithm.
\end{abstract}

\section{INTRODUCCIÓN}

La continuidad en el suministro de energía eléctrica a los centros de consumo (residenciales, comerciales e industriales), en un Sistema Eléctrico de Distribución (SED), en ocasiones puede ser interrumpida por eventos programados (servicios de mantenimiento preventivo) y no programados (contingencias debido a fenómenos climáticos o accidentales) [1]-[2]. La consecuencia de estos eventos es la interrupción del suministro en uno, o varios trechos de carga.

Una vez identificado el, o los trechos, de carga con suministro interrumpido, los interruptores del SED, deben mudar sus estados de operación (interruptores abiertos y cerrados) para obtener una nueva, y óptima, configuración topológica, aislando sólo el, o los trechos, afectados y preservando, en lo posible, la mayor cantidad de carga no afectada [3]. Encontrar la mejor secuencia de maniobras para reconfigurar el SED, de forma empírica, en general insume varias horas de trabajo y pruebas en campo para determinar la mejor solución. En el presente trabajo se propone una metodologia no empirica sino basada en flujo de potencia y la optimizacion de dos objetivos que pretenden mejorar el restablecimiento de la mayor parte de la carga (despues de una contingencia o mantenimiento programado), minimizando a su vez la cantidad de maniobras posibles (la cual reduce el

Digital Object Identifier (DOI):

http://dx.doi.org/10.18687/LACCEI2020.1.1.97

ISBN: 978-958-52071-4-1 ISSN: 2414-6390 tiempo de restablecimiento) [19]. En el presente trabajo, la reconfiguración del SED se realiza a través del Algoritmo Genético (AG) [4], debido a la comprobada eficacia para optimización global de funciones matemáticas que representan sistemas dinámicos reales y complejos. De esa manera, el AG, fue adaptado para la reconfiguración de un SED de topología radial, y busca maximizar la carga a ser restablecida y, de manera simultánea, minimizar el número de maniobras de cierre, o apertura, de interruptores, modificando la configuración inicial del SED.

Para validar la eficiencia del método de restablecimiento implementado, fueron considerados tres sistemas eléctricos de distribución, cada uno con tres alimentadores. Dos SED son de tipo académico y de pequeño porte, y un tercer sistema, de medio porte, proviene de datos reales correspondientes al sistema eléctrico de distribución de la ANDE (Administración Nacional de Electricidad de Paraguay.

\section{FORMULACIÓN DEL PROBLEMA}

El objetivo principal que persigue la reconfiguración, para el restablecimiento del suministro de energía eléctrica, es la maximización de la carga a ser restablecida y la minimización simultánea del número de maniobras de los interruptores (manuales o tele comandados). La reducción en las maniobras de interruptores, está asociada con la reducción en los tiempos de recomposición, evitando reducir la vida útil de esos dispositivos de corte [5].

\section{A. Recomposición de Redes de Distribución}

En la recomposición se pretende el restablecimiento de la energía eléctrica, para la mayor cantidad de consumidores y en el menor tiempo posible, atendiendo todas las restricciones de carga y operación, a través de un método de reconfiguración.

Para el restablecimiento de aquellas áreas, cuyo suministro fue interrumpido, se requiere de una óptima transferencia de alimentación desde las regiones que están energizadas, considerando uno o más alimentadores, con los que el alimentador con el trecho afectado por el evento comparte interruptores de acople (interruptores para transferencia de carga). Esta transferencia, dada a través de maniobras de cierre de interruptores de acople (normalmente abiertos), podrá energizar todos, o parte de aquellos trechos de carga cuyo suministro fue interrumpido, considerando las restricciones de operación y de topología [6].

$18^{\text {th }}$ LACCEI International Multi-Conference for Engineering, Education, and Technology: "Engineering, Integration, and Alliances for a Sustainable Development" "Hemispheric Cooperation for Competitiveness and Prosperity on a Knowledge-Based Economy", 27-31 July 2020, Virtual Edition. 



\section{Lv: Longitud del vector}

- Elitismo. Es utilizado para preservar las mejores soluciones para las próximas generaciones. Con este operador se mantiene la mejor solución encontrada.

El AG es básicamente un método de optimización monoobjetivo, en el cual, se pretende optimizar una única función objetivo (Fitness), y la solución que optimiza dicho objetivo puede tener varias variables de decisión (multimodalidad).

\section{B. Optimización Multi-objetivo}

Son problemas de optimización en los que se pretende optimizar simultáneamente " $n$ " funciones objetivos. Matemáticamente, se representan de la siguiente manera:

$$
\operatorname{Max} / \operatorname{Min} f_{n}(x)
$$

donde $\mathrm{fn}(\mathrm{x})$ es el vector de funciones objetivo (Fitness)

\section{Adecuación del AG para Optimización Multi-Objetivos}

Actualmente, existen múltiples técnicas para adecuar el AG para problemas multi-objetivos [8], [9], [10] y [11]. En el presente trabajo fue implementado el método de suma ponderada de objetivos, el cual consiste en calcular un Fitness basado en la combinación lineal de las funciones matemáticas, que representan los objetivos que se desean optimizar. La expresión matemática del método de suma ponderada queda resumida en la ecuación (9):

$$
F(x)=a_{1} \cdot F_{1}(x)+a_{2} \cdot F_{2}(x)+\cdots+a_{n} \cdot F_{n}(x)
$$

donde $F(x)$ representa al Fitness que el AG debe optimizar; y, $a_{1}, a_{2}, \ldots, a_{n}$, corresponden a los coeficientes de la suma ponderada de las " $n$ " funciones objetivos.

\section{Iv. METODOLOGÍA PROPUESTA}

El método propuesto de reconfiguración para maximizar el restablecimiento de la energía está basado en la adecuación del AG para optimización multi-objetivo, descrito por medio de la ecuación (9). Además, fueron desarrollados cuatro algoritmos complementarios para el cálculo de las funciones objetivos y para satisfacer la condición de radialidad del SED bajo estudio, y que son Nueva topología, Radialidad, Transferencia de Carga y Flujo de potencia.

\section{A. Nueva Topología}

Este algoritmo realiza un análisis de la topología del SED, y determina una lista de interruptores disponibles para ser abiertos, del lado de la fuente y del lado de la carga, para aislar el trecho con falla. Este procedimiento minimiza el área afectada en función de la disponibilidad y la conveniencia de maniobras. Además, el referido algoritmo, indica a través de una lista, cuales son los interruptores disponibles para maniobras de restablecimiento. La lista de interruptores establece un vector de alternativas de solución. Cada vector solución está conformado por valores binarios 1 y 0 , donde el número 1 indica que el interruptor está cerrado y el número 0 asocia un interruptor abierto. Los referidos estados de los interruptores establecen la configuración de todo el SED. La Fig. 1, muestra un ejemplo de un SED con tres alimentadores, representados por su vector solución. El AG obtiene, en cada generación, una población de $\mathrm{N}$ vectores factibles.

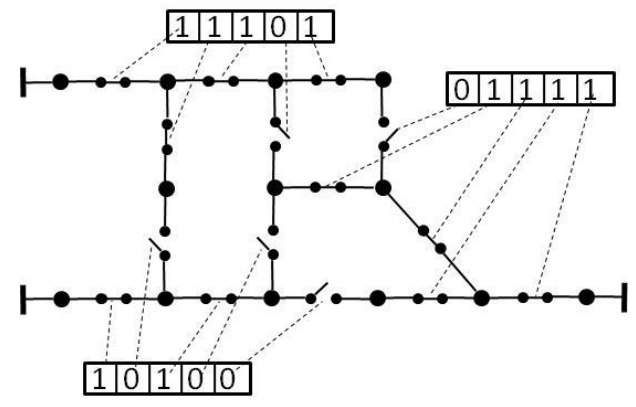

Fig. 1 Conformación de vectores solución.

El AG recibe el listado de seccionadores, determinado anteriormente en función al evento localizado en un trecho específico, y aplica "Tabla Verdad" que genera otra tabla conteniendo todas las posibles combinaciones del estado de los interruptores, y de las topologías, para el vector solución. Con la referida tabla determinada, se genera la población inicial para el AG, logrando una substancial reducción en el tiempo computacional, para establecer las soluciones iniciales. Cada vector solución debe ser verificado, para comprobar si la topología es efectivamente radial. Luego, de verificar la radialidad de la topología, se establece la transferencia de carga permitida para dicha configuración, y seguidamente, se aplica el algoritmo de Flujo de Potencia, para el cálculo de todas las funciones objetivos, y después, se realiza el cálculo del correspondiente Fitness. Seguidamente, después del cálculo del Fitness, para cada individuo de la población, el AG, en cada generación, aplica los operadores de evolución (Selección o reproducción) y genéticos (Cruzamiento y Mutación) [4], para la obtención de nuevos individuos, hasta que el criterio de parada (máximo número de generaciones) sea satisfecho. A su vez, a través del Elitismo [4], es evaluado en cada generación el individuo con el Fitness más alto obtenido, con la finalidad de preservarlo para las siguientes generaciones.

\section{B. Radialidad}

En este algoritmo se realiza un análisis de la topología del SED, utilizando la Teoría de Grafos [12], y la técnica de ordenamiento de redes descripto en [13]. Solo aquellas topologías confirmadas como radiales continúan el proceso. Si un vector de solución representa una topología no radial, se ejecuta automáticamente un algoritmo de corrección de radialidad que analiza la topología que resulta de aplicar el vector solución y despeja la causa que ocasiona la no radialidad. La corrección de radialidad asegura la capacidad de programa computacional de analizar sistemas eléctricos de 
gran porte, donde los espacios de búsqueda crecen exponencialmente. Luego de que se asegura que el vector solución es radial, dicho vector es enviado a la función de Transferencia de Carga.

\section{Transferencia de Carga}

Con la transferencia de carga se modifica la base de datos en función de las maniobras, de cierre y abertura de interruptores, y se reasignan los correspondientes trechos y cargas a los recursos, adoptados en cada vector de solución. El procedimiento de transferencia de carga se realiza solamente sobre aquellos vectores solución que representan topologías radiales; y, se transfieren las cargas al alimentador o los alimentadores de apoyo, mientras dure el evento programado o no programado que afectó al referido trecho, y la desconexión de las cargas asociadas al trecho. Como resultado de esta transferencia, se reorganiza la base de datos del SED que se transfiere a la función de Flujo de Potencia.

\section{Flujo de Potencia}

Las redes trifásicas fueron consideradas simétricas y equilibradas, y su resolución fue considerando los parámetros de secuencia positiva y la representación monofásica de la red. Esta alternativa fue tenida en cuenta porque el desequilibrio de carga entre los alimentadores del SED, de la ANDE (Administración Nacional de Electricidad del Paraguay), considerados para el estudio, es menor al 1,5\%, y por consiguiente, la representación monofásica es válida. De esa manera, fue implementado un algoritmo de Flujo de Potencia adecuado para sistemas radiales, adoptando un modelo monofásico de barredura inversa/directa, de sumas de corrientes [14], y utilizando el modelo de línea corta para redes primarias y secundarias, y empleando la representación de potencia constante para las cargas [13], [17].

\section{E. Funciones Objetivos}

Como el objetivo de la reconfiguración, para el restablecimiento, consiste en determinar la máxima transferencia de carga, con el menor número posible de maniobras, el procedimiento para el cálculo de las funciones objetivo es:

-- Potencia transferida. A partir de los resultados del flujo de potencia, se sigue el siguiente procedimiento de cálculo:

- Se calcula la potencia demandada por el SED.

$$
\begin{aligned}
& P_{b e}=\sum_{i \in B_{T}} P_{i} \\
& Q_{b e}=\sum_{i \in B_{T}} Q_{i}
\end{aligned}
$$

donde:

$P_{b e}:$ Potencia activa instalada antes del evento.

$Q_{b e}$ : Potencia reactiva instalada antes del evento.

$P_{i} \quad$ : Potencia activa total en el nodo $i$.

$Q_{i} \quad$ : Potencia reactiva total en el nodo $i$.
$B_{T} \quad$ : Conjunto de nodos de carga.

- Se calcula la potencia interrumpida en el SED.

$$
\begin{aligned}
& P_{\text {int }}=\sum_{i \in B_{T}} P_{i}^{f} \\
& Q_{\text {int }}=\sum_{i \in B_{T}} Q_{i}^{f}
\end{aligned}
$$

donde:

$P_{\text {int }}$ : Potencia activa total interrumpida.

$Q_{\text {int }}$ : Potencia reactiva total interrumpida.

$P_{i}^{f}$ : Potencia activa interrumpida en el nodo $i$.

$Q_{i}^{f}$ : Potencia reactiva interrumpida en el nodo $i$.

$B_{T}$ : Conjunto de nodos de carga

- Se calcula la potencia instalada en el SED, luego de la transferencia de carga.

$$
\begin{aligned}
& P_{a t}=\sum_{i \in B_{T}} P_{i}^{a} \\
& Q_{a t}=\sum_{i \in B_{T}} Q_{i}^{a}
\end{aligned}
$$

donde:

$P_{a t}:$ Potencia activa luego de la transferencia.

$Q_{a t}$ : Potencia reactiva luego de la transferencia.

$P_{i}^{a}$ : Potencia activa en nodo $i$ luego de transferir.

$Q_{i}{ }^{a}$ : Potencia reactiva en el nodo $i$ luego de transferir.

$B_{T}$ : Conjunto de nodos de carga

- Se calcula la potencia no restablecida en el SED luego del evento.

$$
\begin{aligned}
& P_{n r}=P_{b e}-P_{a t} \\
& Q_{n r}=Q_{b e}-Q_{a t}
\end{aligned}
$$

donde:

$P_{n r}$ : Potencia activa no restablecida luego de la transferencia.

$Q_{n r}$ : Potencia reactiva no restablecida luego de la transferencia

- Se calcula la potencia restablecida en el SED con la transferencia de carga.

$$
\begin{aligned}
& P_{r}=P_{\text {int }}-P_{n r} \\
& Q_{r}=Q_{\text {int }}-Q_{n r} \\
& S_{r}=\sqrt{\left(P_{r}^{2}+Q_{r}^{2}\right)}
\end{aligned}
$$

donde:

$S_{r}$ : Potencia aparente restablecida con la transferencia.

La función objetivo $f_{1}$ es igual a la potencia restablecida $S_{r}$.

$$
f_{1}(\mathrm{x})=S_{r}
$$

--Número de Maniobras. Mediante un contador se enumera la cantidad de interruptores cuyos estados mudan (de abierto para cerrado, y viceversa).

$$
f_{2}(\mathrm{x})=\sum_{k=1}^{N C+N A} C E c h_{k}
$$


El símbolo $C E c h_{k}$, en la ecuación (22), está indicando el cambio de estado del interruptor k; de cerrado para abierto o viceversa, entonces se contabiliza en 1 , y 0 en caso contrario. Entonces, de la cantidad total de interruptores, establecido por la suma de interruptores normalmente cerrado (NC) más el número de interruptores normalmente abierto (NA), se contabiliza aquellos que cambiaron sus respectivos estados.

Luego de la obtención de las funciones objetivos, asociado a cada vector solución (topología), se procede al análisis del Fitness, teniendo en cuenta la expresión matemática (9), representando por medio de la ecuación (23).

$$
F(x)=a_{1} \cdot F_{1}(x)+a_{2} \cdot F_{2}(x)
$$

La ecuación (23) corresponde a la suma ponderada de las funciones $F_{1}(x)$ y $F_{2}(x)$, donde $x$ representa el vector solución, tal como se ilustra en la Fig. 1. Con la finalidad de normalizar el Fitness, se considera que el valor límite para $F_{1}(x)$ es $F 1$ max, y el valor límite para $F_{2}(x)$ es $F 2$ min. $F 1$ max adopta el valor de $100 \mathrm{MVA}$, que es la potencia base (MVA) del SED, y $F 2$ min adopta el valor 1 , que es el mínimo número de maniobras esperado. Para balancear los resultados de $F_{1}(x)$ con los de $F_{2}(x)$, se aplica el factor $F n$. De esta forma, los valores límites se relacionan de la siguiente forma.

$$
F(x)=a_{1} \cdot \frac{F n \times F_{1}(x)}{F 1 \max }+a_{2} \cdot \frac{F 2 \min }{F_{2}(x)}
$$

donde:

$F_{1}(x)$ : Es el valor total de la potencia restablecida;

$F_{2}(x)$ : Es la cantidad de maniobras realizadas;

F1max: Es el máximo valor de potencia restablecida, en una iteración (generación);

F2min: Es el mínimo número de maniobras, en una determinada generación;

$F(\mathrm{x})$ : Es el Fitness, para cada $\mathrm{x}$, que se optimiza.

Considerando el valor mínimo de $F_{1}(x)=100 \mathrm{kVA}$ y $F 1$ max $=100 \mathrm{MVA}$, al dividirlos bajo la misma unidad de medida se obtiene:

$$
\frac{F_{1}(x)}{F_{1} \max }=\frac{100.000}{100.000 .000}[V A]=0,001
$$

Multiplicando cada expresión por 1.000 para normalizar el numerador con el denominador, en el peor caso (mínimo valor de $f_{1}(x)$ ), se obtiene como resultado el siguiente valor:

$$
1.000 \times \frac{F_{1}(x)}{F_{1} \max }=1.000 \times \frac{100.000}{100.000 .000}[V A]=1
$$

De esta manera, el máximo valor para el primer sumando es igual a la unidad, y el máximo valor del segundo sumando es también igual a 1; por lo tanto, el máximo valor de la función $F(x)=2$, cuando la función $F_{1}(x)$ es mínimo. Por otro lado, cuando la función $F_{1}(x)$ es máximo, es decir asume valores elevados, siendo el máximo valor posible de $F_{1}(x)=100 \mathrm{MVA}$, el primer sumando de la función $F(x)$ es igual a 1000, y el máximo del segundo sumando continuaría siendo
1, por lo tanto, el máximo valor de la función $F(x)=1001$. Esta solución garantiza, en todos los casos posibles, el escalonamiento adecuado de los valores del fitness.

Los valores numéricos adoptados para los coeficientes de ponderación, $a_{1}$ y $a_{2}$, son iguales a 1 para que ambas funciones objetivos, $F_{1}(x)$ y $F_{2}(x)$, posean el mismo grado de importancia en la búsqueda de una única solución que satisfaga simultáneamente ambas condiciones.

\section{F. Flujograma}

En la Fig.2 se presenta el flujograma del algoritmo implementado, resumiendo las partes principales que fueron consideradas para la solución del presente trabajo, y que facilitó la estructuración y la secuencia de cálculos.

En el cuadro "Tramo con Evento" se informa al algoritmo el trecho en falta y en el cuadro "Aislación del Tramo" corre de forma automática una función que determina la lista de interruptores y sus correspondientes estados de abierto y cerrado, generando una nueva topología, que permita separar el trecho con falta del resto de los trechos, tal como fue descripto en la Sección IV para la función Nueva Topología, del algoritmo de reconfiguración implementado.

Seguidamente, se verifica la radialidad de cada una de las nuevas topologías obtenidas. En el caso que la topología sea radial, se realiza el cálculo de la potencia transferida, y a continuación se aplica el Flujo de Potencia, para determinar el valor de las funciones objetivos $F_{1}(x)$ y $F_{2}(x)$, con los que finalmente se calcula el Fitness. Este procedimiento se realiza con cada individuo (nueva topología) obtenido. Después, se aplica el AG para obtener nuevas y mejores topologías (valor de $F(x)$, de la ecuación (24), cada vez más alto), hasta alcanzar el criterio de parada.

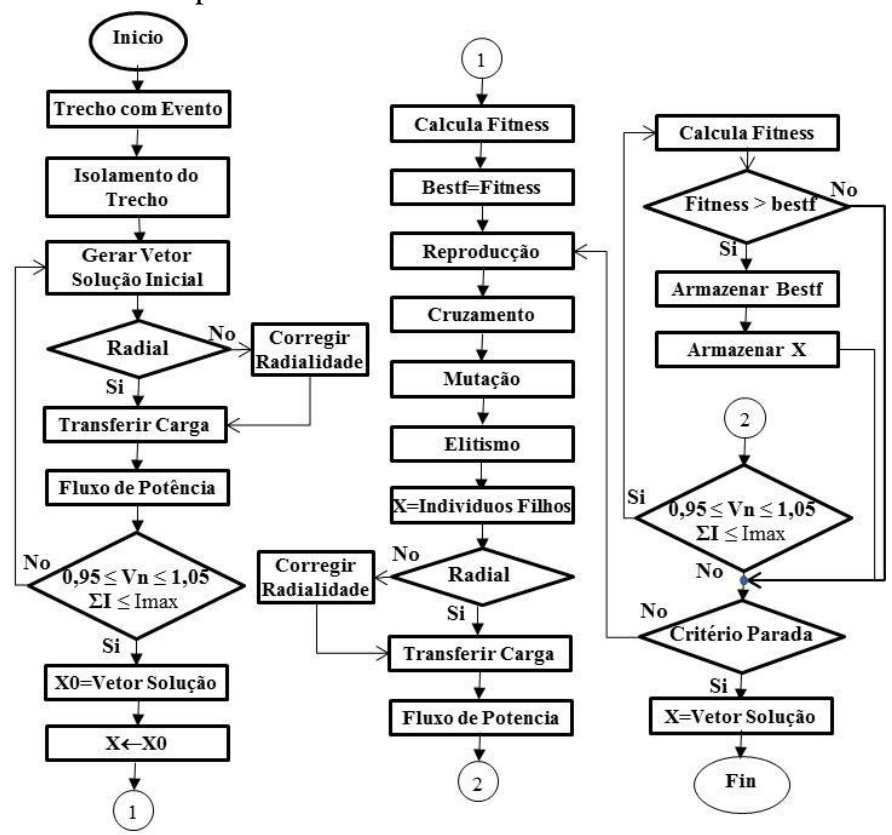

Fig. 2 Flujograma del Algoritmo de Reconfiguración. 


\section{v. SIMULACIÓN Y ANÁLISIS DE RESULTADOS}

\section{A. Ambiente Computacional}

El MatLab® fue utilizada para la implementación de los algoritmos del método de recomposición propuesto, en un computador con Intel Core i5-3470, 3,2 GHz e 4 GB RAM.

\section{B. Sistemas de Prueba}

Para validar la eficiencia del algoritmo de reconfiguración propuesto, se consideró los siguientes sistemas de prueba.

Sistema 1.Es un SED de pequeño porte, modificado a partir de un esquema unifilar propuesto en [13], que sirvió de base para validar el algoritmo de flujo de potencia. Este SED, que inicialmente contenía un alimentador sin recursos, fue adicionado con dos alimentadores, de similares características, e interruptores para transferencias de carga, quedando integrado con 29 nodos de carga y 26 interruptores, siendo 18 normalmente cerrados (NC) y 8 normalmente abiertos (NA), según la Fig.3.

Sistema 2.Es un SED de pequeño porte elaborado a partir de un alimentador de la India [16]. Se agregaron dos alimentadores, y recursos para transferencias de carga, con 42 nodos de carga y 25 seccionadores (18 NC y 7 NA), conforme se ilustra en la Fig. 4.

Sistema 3.Es un SED de medio porte, perteneciente al sistema eléctrico de la ANDE [18], y está compuesto por tres alimentadores de la Subestación Tres Bocas (TBO1, TBO2 y TBO8), con 221 nodos de carga, 26 interruptores, (19 NC y 7 NA), según la Fig. 5.

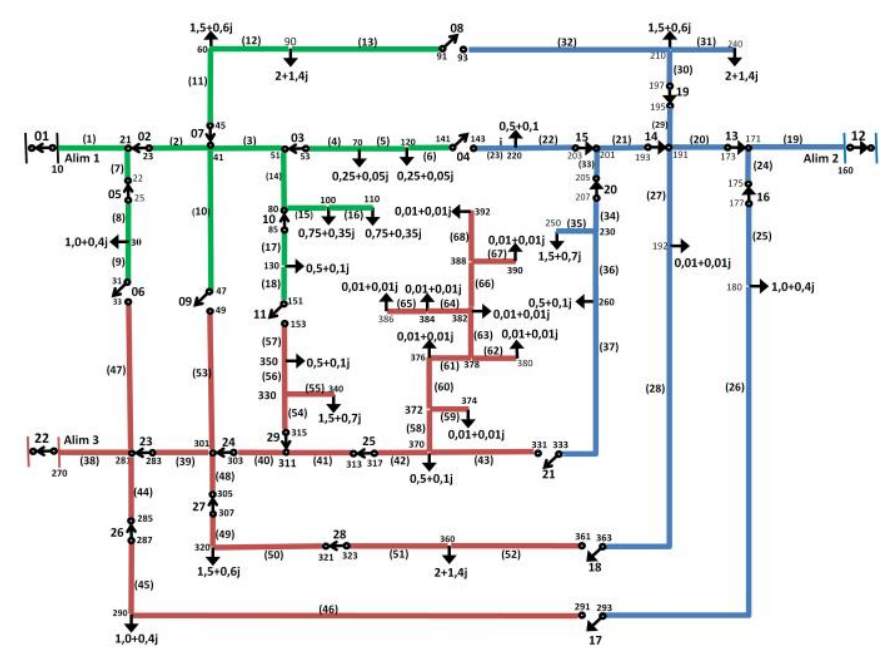

Fig. 3 Sistema Eléctrico de Distribución $\mathrm{N}^{\circ} 1$.

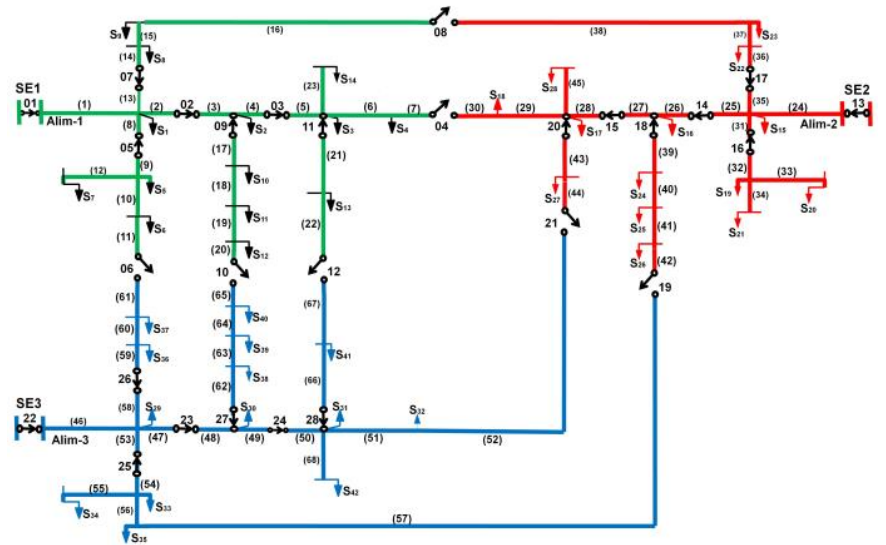

Fig. 4 Sistema Eléctrico de Distribución $N^{\circ} 2$.

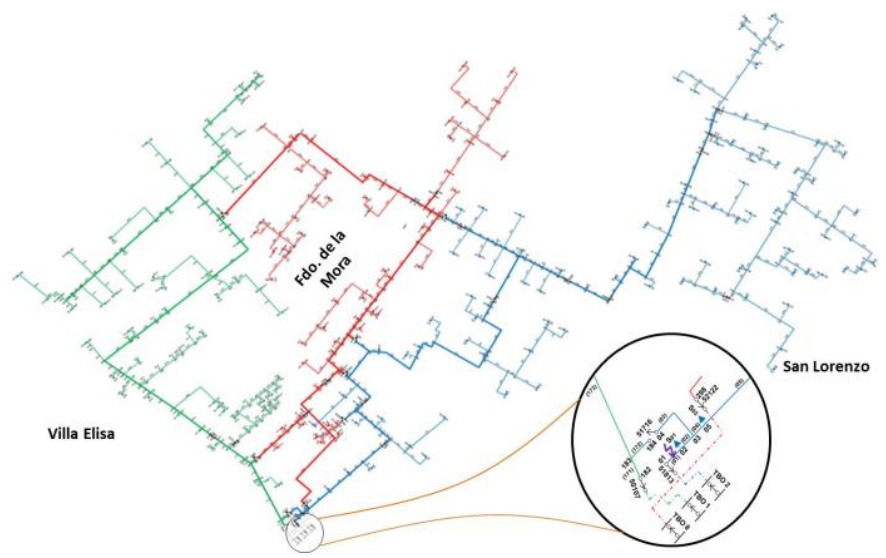

Fig. 5 Sistema Eléctrico de Distribución $N^{\circ} 3$.

\section{Resultados Obtenidos}

- Para el Sistema 1:

En la Tabla I se presentan las condiciones iniciales antes de la simulación de una falla en el Tramo 1, correspondiente al trecho $10-21$, en el alimentador 1 :

TABLE I

CONDICIONES ANTES DEL EVENTO

\begin{tabular}{|l|c|c|c|}
\hline \multirow{2}{*}{} & \multicolumn{3}{|c|}{ Alimentador } \\
\cline { 2 - 4 } & 1 & 2 & 3 \\
\hline Tensión Nominal (kV) & 13,8 & 13,8 & 13,8 \\
\hline Carga Instalada (MVA) & $7+j \cdot 3,3$ & $7,01+j \cdot 3,31$ & $7,08+j \cdot 3,38$ \\
\hline Potencia Entregada (MVA) & $7,08+j \cdot 3,44$ & $7,10+j \cdot 3,45$ & $7,17+j \cdot 3,53$ \\
\hline Corriente Total (A) & 322,98 & 323,82 & 0,0783 \\
\hline Factor de Potencia & 0,90 & 0,90 & 0,90 \\
\hline Pérdidas Totales en la Red (MVA) & $0,09+j \cdot 0,15$ & $0,09+j \cdot 0,14$ & $0,08+j \cdot 0,14$ \\
\hline Tensión Mínima (kV) & 13,794 & 13,794 & 13,790 \\
\hline Corriente Máxima Admisible (A) & 650 & 650 & 650 \\
\hline
\end{tabular}

En la Tabla II se describe la parametrización del AG para la simulación de la reconfiguración del Sistema 1. 
TABLE II

CONFIGURACIÓN DEL AG

\begin{tabular}{|l|c|l|c|}
\hline Población Inicial & 20 & Cantidad de Generaciones & 30 \\
\hline Probabilidad de Cruzamiento & 0,7 & Cantidad de Genes & 2 \\
\hline Probabilidad de Mutación & 0,013 & & \\
\hline
\end{tabular}

Los resultados de la reconfiguración, para restablecer la carga, luego de simular un evento en el Tramo 1 se muestran en la Tabla III. Se gastó un tiempo promedio de simulación de 33,55s, obtenido luego de realizar la simulación cinco veces.

TABLE III CONFIGURACIÓN LUEGO DEL EVENTO

\begin{tabular}{|c|c|c|c|}
\hline & \multicolumn{3}{|c|}{ Alimentador } \\
\hline & 1 & 2 & 3 \\
\hline Potencia Interrumpida (MVA) & $7+j \cdot 3,3$ & & \\
\hline \multicolumn{4}{|l|}{ Aislación } \\
\hline Lado Fuente: Abrir Seccionador & 1 & & \\
\hline Lado Carga: Abrir Seccionador & $2-5$ & & \\
\hline Interruptores Operables & \multicolumn{3}{|c|}{$7-9-3-10-8-4-11-6$} \\
\hline Fitness & \multicolumn{3}{|c|}{77,5} \\
\hline \multicolumn{4}{|l|}{ Reconfiguración } \\
\hline \multicolumn{4}{|l|}{ Abrir Interruptor } \\
\hline Cerrar Interruptor & $8-6$ & & \\
\hline Tensión Nominal (kV) & 13,8 & 13,8 & 13,8 \\
\hline Carga Instalada (MVA) & 0 & $13,01+j \cdot 6,21$ & $8,08+j \cdot 3,78$ \\
\hline Potencia Entregada (MVA) & 0 & $13,33+j \cdot 6,76$ & $8,18+j \cdot 3,95$ \\
\hline Corriente Total (A) & 0 & 612,81 & 418,8 \\
\hline Factor de Potencia & 0 & 0,89 & 0,90 \\
\hline Pérdidas Totales en la Red (MVA) & 0 & $0,32+j \cdot 0,52$ & $0,10+j \cdot 0,17$ \\
\hline Tensión Mínima (kV) & 13,794 & 13,495 & 13,77 \\
\hline Carga Restablecida (MVA) & \multicolumn{3}{|c|}{$7+j \cdot 3.3$} \\
\hline Porcentaje de Carga Restablecida (\%) & \multicolumn{3}{|c|}{$100 \%$} \\
\hline Tiempo Promedio de Resolución (s) & \multicolumn{3}{|c|}{33,55} \\
\hline
\end{tabular}

- Para el Sistema 2:

En la Tabla IV se muestra las condiciones iniciales antes de la simulación de una falla en el Tramo 24, en el alimentador 2.

TABLE IV

CONFIGURACIÓN ANTES DEL EVENTO

\begin{tabular}{|l|c|c|c|}
\hline \multirow{2}{*}{} & \multicolumn{3}{|c|}{ Alimentador } \\
\cline { 2 - 4 } & 1 & 2 & 3 \\
\hline Tensión Nominal (kV) & 11 & 11 & 11 \\
\hline Carga Instalada (MVA) & $1,23+j \cdot 1,25$ & $1,23+j \cdot 1,25$ & $1,23+j \cdot 1,25$ \\
\hline Potencia Entregada (MVA) & $1,29+j \cdot 1,31$ & $1,29+j \cdot 1,31$ & $1,29+j \cdot 1,31$ \\
\hline Corriente Total (A) & 96,57 & 96,57 & 96,57 \\
\hline Factor de Potencia & 0,70 & 0,70 & 0,70 \\
\hline Pérdidas Totales en la Red (MVA) & $0,06+j \cdot 0,06$ & $0,06+j \cdot 0,06$ & $0,06+j \cdot 0,06$ \\
\hline Tensión Mínima (kV) & 10,390 & 10,390 & 10,390 \\
\hline Corriente Máxima Admisible (A) & 150 & 150 & 150 \\
\hline
\end{tabular}

En la Tabla V se describe la parametrización del AG para la simulación de la reconfiguración del Sistema 2.

TABLE V

CONFIGURACIÓN DEL AG

\begin{tabular}{|l|c|l|c|}
\hline Población Inicial & 20 & Cantidad de Generaciones & 30 \\
\hline Probabilidad de Cruzamiento & 0,7 & Cantidad de Genes & 2 \\
\hline Probabilidad de Mutación & 0,014 & & \\
\hline
\end{tabular}

En la Tabla VI se presentan los resultados de la reconfiguración, y el tiempo promedio de computación, que alcanzó 38,5s, obtenido luego de realizar la simulación cinco veces.

TABLE VI

CONFIGURACIÓN LUEGO DEL EVENTO

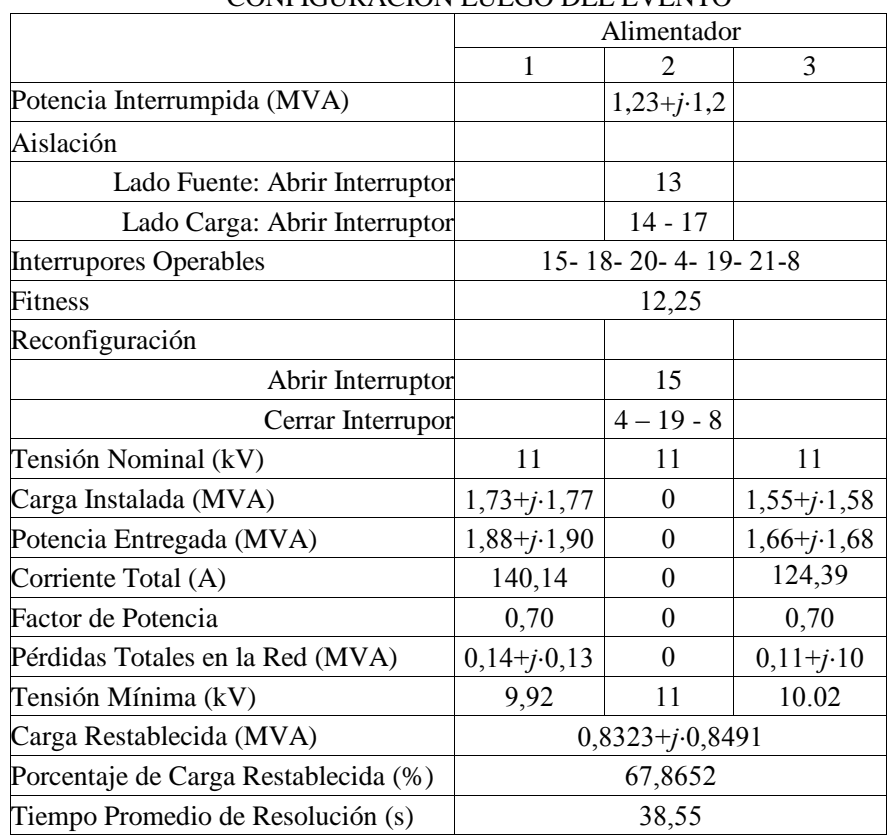

- Para el Sistema 3:

En la Tabla VII se describe las condiciones iniciales antes de la simulación de una falla en el Tramo 1, en el alimentador TBO-2.

TABLE VII CONFIGURACIÓN ANTES DEL EVENTO

\begin{tabular}{|l|c|c|c|}
\hline \multirow{2}{*}{} & \multicolumn{3}{|c|}{ Alimentador } \\
\cline { 2 - 4 } & TBO-2 & TBO-1 & TBO-8 \\
\hline Tensión Nominal (kV) & 23 & 23 & 23 \\
\hline Carga Instalada (MVA) & $8,56+j \cdot 4,08$ & $8,34+j \cdot 4,01$ & $5,06+j \cdot 2,46$ \\
\hline Potencia Entregada (MVA) & $8,77+j \cdot 4,27$ & $8,39+j \cdot 4,08$ & $5,09+j \cdot 2,49$ \\
\hline Corriente Total (A) & 244,75 & 234,2 & 142,08 \\
\hline Factor de Potencia & 0,90 & 0,90 & 0,90 \\
\hline Pérdidas Totales en la Red (MVA) & $0,2+j \cdot 0,19$ & $0,06+j \cdot 0,07$ & $0,03+j \cdot 0,03$ \\
\hline Tensión Mínima (kV) & 22,112 & 22,68 & 22,788 \\
\hline Corriente Máxima Admisible (A) & 360 & 360 & 360 \\
\hline
\end{tabular}

En la Tabla VIII se muestra la parametrización del AG para la simulación de la reconfiguración del Sistema 3.

TABLE VIII

CONFIGURACIÓN DEL AG

\begin{tabular}{|l|c|l|c|}
\hline Población Inicial & 20 & Cantidad de Generaciones & 30 \\
\hline Probabilidad de Cruzamiento & 0,7 & Cantidad de Genes & 2 \\
\hline Probabilidad de Mutación & 0,013 & & \\
\hline
\end{tabular}

En la Tabla IX se presentan los resultados de la reconfiguración, y el tiempo promedio de computación, que alcanzó 73,4s, obtenido luego de realizar la simulación cinco veces. 
TABLE IX

CONFIGURACIÓN LUEGO DEL EVENTO

\begin{tabular}{|c|c|c|c|}
\hline & \multicolumn{3}{|c|}{ Alimentador } \\
\hline & TBO-2 & TBO-1 & TBO-8 \\
\hline Potencia Interrumpida (MVA) & $8,562+j \cdot 4,084$ & & \\
\hline \multicolumn{4}{|l|}{ Aislación } \\
\hline Lado Fuente: Abrir Interruptor & 51813 & & \\
\hline Lado Carga: Abrir Interruptor & 53003 & & \\
\hline Interruptores Operables & \multicolumn{3}{|c|}{$\begin{array}{c}52576-51654-52341-52342-52343- \\
50117-52208-50120-52209-50186- \\
50187-53723-53718\end{array}$} \\
\hline Fitness & \multicolumn{3}{|c|}{82,3333} \\
\hline \multicolumn{4}{|l|}{ Reconfiguración } \\
\hline \multicolumn{4}{|c|}{ Abrir Interruptor $51654-53718$} \\
\hline Cerrar Interruptor & 50120 & & \\
\hline Tensión Nominal (kV) & 23 & 23 & 23 \\
\hline Carga Instalada (MVA) & 0 & $8,34+j \cdot 4,01$ & $12,43+$ \\
\hline Potencia Entregada (MVA) & 0 & $8,39+j \cdot 4,08$ & $12,73+j \cdot 6,2$ \\
\hline Corriente Total (A) & 0 & 234,2 & 356,2 \\
\hline Factor de Potencia & - & 0,90 & 0,90 \\
\hline Pérdidas Totales en la Red (MVA) & 0 & $0,06+j \cdot 0,07$ & $0,30+j \cdot 0,31$ \\
\hline Tensión Mínima (kV) & 23 & 22,68 & 22,011 \\
\hline Carga Restablecida (MVA) & \multicolumn{3}{|c|}{$7,3659+j \cdot 3.5135$} \\
\hline Porcentaje de Carga Restablecida (\%) & \multicolumn{3}{|c|}{86,03} \\
\hline Tiempo Promedio de Resolución (s) & \multicolumn{3}{|c|}{73,4} \\
\hline
\end{tabular}

\section{Evaluación de los Resultados de Simulación}

Luego de la ocurrencia de una falla y la identificación del trecho afectado, el objetivo principal de toda concesionaria de energía es la de aislar el trecho afectado, y restaurar la carga del sistema, en su totalidad o la máxima posible, en el menor tiempo posible.

En ese contexto, con el algoritmo de reconfiguración propuesto, se determinaron para maniobras, en los Sistemas 1 y 2, tres interruptores, y en el Sistema 3, la maniobra de dos interruptores, para aislar el trecho afectado. A su vez, en el Sistema 1 se determinó el cierre de 2 interruptores de acoplamiento y en los Sistemas 2 y 3, se estableció la maniobra de tres interruptores, para la transferencia de carga. Por lo tanto, el número de interruptores maniobrados para aislar el trecho afectado, y, luego, permitir la transferencia de potencia, para restablecimiento de la carga, se mantuvo como máximo en tres, en ambos casos. En todos los casos, luego de la reconfiguración, el perfil de tensión en todo el sistema se mantuvo dentro de los valores permitidos. Es decir, no hubo sobretensión ni subtensión.

En el Sistema 1, para el evento simulado, se consiguió restablecer toda la carga. En cambio, para los Sistemas 2 y 3, no fue posible el restablecimiento de toda la carga. Sin embargo, se obtuvo un porcentaje de restablecimiento por encima del 60\% (67,8\% para el Sistema 2 y $86 \%$ para el Sistema 3), principalmente por la baja capacidad de transferencia de los interruptores de acoplamiento asociados.

\section{CONCLUSIONES}

La reconfiguración propuesta, utilizando el AG, logró demostrar su efectividad en los tres sistemas eléctricos considerados, obteniendo el mínimo número de interruptores a ser maniobrados para aislar el trecho en falta y maximizar la transferencia de potencia, de tal manera a permitir que se restablezcan las cargas entre el $68 \%$ para el Sistema 2 y el 100\% para el Sistema 1.

Además, las tensiones y las corrientes, en todo el sistema, se mantienen dentro del rango admisible. El tiempo de computación, utilizado por el algoritmo de reconfiguración propuesto, encuentra la topología óptima en un tiempo entre 33s para sistemas de pequeño porte y 73s para sistemas de medio porte, que son valores reducidos y razonables para análisis de tiempo real, tal que puede ser aprovechado como herramienta de ayuda para despacho de carga, o también, para planificación de la operación de sistemas.

\section{REFERENCIAS}

[1] John Peñaloza ; Jairo Yumbla ; Julio López ; Antonio Padilha-Feltrin, (2019). "Optimal Distribution Network Reconfiguration with Distributed Generation using a Genetic Algorithm”. Published in: 2019 IEEE PES Innovative Smart Grid Technologies Conference - Latin America (ISGT Latin America).

[2] A. Zidan, M. Khairalla, A. M. Abdrabou, T. Khalifa, K. Shaban, A. Abdrabou, R. E. Shatshat, A. M. Gaouda (2017). "Fault Detection, Isolation, and Service Restoration in Distribution Systems: State-ofthe-Art and Future Trends". IEEE Transaction on Smart Grid, Vol. 8, No. 5, pp. 2170 - 2185, September 2017.

[3] A. S. Abubakar, K. R. Ekundayo, A. A. Olaniyan (2019). "Optimal reconfiguration of radial distribution networks using improved genetic algorithm". Proceedings of Nigerian Journal of Technological Development, Vol $16 \mathrm{~N}^{\circ} 1,2019$.

[4] Goldberg, David E. (1989). "Genetic Algorithms in Search, Optimization, and Machine Learning”. 1st Ed. Addison-Wesley Longman Publishing Co., Inc. Boston, MA, USA.

[5] J. B. Leite, J. R. Sanches (2017), "Development of a Self-Healing Strategy with Multiagent systems for Distribution Networks". IEEE Transaction on Smart Grid, Vol. 8, No. 5, pp. 2198 - 2206, September 2017.

[6] G. Eason, B. Noble, and I.N. Sneddon, "On certain integrals of Lipschitz-Hankel type involving products of Bessel functions," Phil. Trans. Roy. Soc. London, vol. A247, pp. 529-551, April 1955. (references).

[7] Lee, S-J., Lim, S-I., Ahn, B-S (1997). "Service Restoration of Primary Distribution Systems Base on Fuzzy Evaluation of Multi-Criteria”. IEEE Power Engineer Society Pre-print. PE-701-PWRS - 0.

[8] Ramadoni Syahputra (2017). "Distribution Network Optimization Based on Genetic Algorithm". Journal of Electrical Technology UMY.

[9] Ahmed Tiguercha; Ahmed Amine Ladjici; Mohamed Boudour (2017). "Optimal radial distribution network reconfiguration based on multi objective differential evolution algorithm". Published in: 2017 IEEE Manchester PowerTech.

[10] N. Arputha Aravinth ; Vikas Anand Vatul ; K. Narayanan. (2019). "A Multi Objective Framework for Optimal Installation of Distributed Generator Units Using Genetic Algorithm”. Published in: 2019 IEEE Innovative Smart Grid Technologies - Asia (ISGT Asia). 
[11] Priyanka Gangwar; Sri Niwas Singh; Saikat Chakrabarti (2019). "Multi-objective planning model for multi-phase distribution system under uncertainty considering reconfiguration". Journals \& magazines, IET Renewable Power Generation, Volume 13, Issue 12.

[12] Usharani Raut; Sivkumar Mishra (2017). "Power distribution network reconfiguration for loss minimization using a new graph theory based genetic algorithm". Published in: 2017 IEEE Calcutta Conference (CALCON).

[13] Kagan, N.; Oliveira, C. C. B. de; Robba, E. J. (2005). "Introdução aos Sistemas de Distribuição de Energía Elétrica". São Paulo: Edgard Blucher.

[14] Amasifen, J. C. (2003). "Algoritmo evolutivo dedicado à solução do problema de reconfiguração de sistemas de distribuição radiais". 184f. Disertação (Mestrado em Engenharia Elétrica) - Faculdade de Engenharia. Universidade Estadual Paulista, Ilha Solteira.

[15] Castaño, Samuel Ramirez (2004). "Redes de Distribución de Energía". Universidad Nacional de Colombia. Tercera Edición.

[16] Das, D., Kothari, D.P., Kalam, A.. "Simple and efficient method for load flow solution of radial distribution networks". Electrical Power \& Energy Systems, Vol. 17, No. 5, pp 335-346. 1995.

[17] Olascoaga Durán, Jaime (2018) “Aplicación en tiempo real de técnicas de cálculo de flujo de cargas en redes de distribución". Máster Universitario en Ingeniería de Minas. http://hdl.handle.net/10651/48000.

[18] CYMDIST-CYME 7.0 Rev. 6. Sistema Informático de Simulación de Distribución y Base de Datos de Distribución.

[19] Cáceres, N., Chaparro, E., Barán, B., "Reconfiguración Determinística de Sistemas Eléctricos de Distribución radiales basado en Bloques Lineales". Seminario del Sector Eléctrico Paraguayo - XII SESEP, Asunción, Paraguay, 2016. https://www.cigre.org.py/index.php/201403-19-13-47-38/blog 